\title{
Non-Dipping Blood Pressure Variations in Juvenile Kazakhs are Derived from Increased Sympathetic Activity and Decreased Parasympathetic Activity Particularly Decreased Daytime Physical Activity
}

\author{
Hiroshi Kawamura ${ }^{1 *}$, Yukio Ozawa ${ }^{2}$, Yoichi Izumi ${ }^{3}$, Yuji Kasamaki ${ }^{4}$, Tomohiro Nakayama ${ }^{5}$, \\ Hiromi Mitsubayashi ${ }^{6}$ and Yuhei Ichimaru ${ }^{7}$ \\ ${ }^{1}$ Blood Pressure Center, Myojyougakuen (MJG) Cardiovascular Institute, Japan \\ ${ }^{2}$ Department of Cardiology, MJG Cardiovascular Institute, Japan \\ ${ }^{3}$ Department of Cardiology, Kanazawa Medical University, Japan \\ ${ }^{4}$ Department of General Medicine, Nihon University School of Medicine, Japan \\ ${ }^{5}$ Department of Laboratory Medicine, Nihon University School of Medicine, Japan \\ ${ }^{6}$ Department of Medicine, Nippon Dental University, Japan \\ ${ }^{7}$ Department of Nutrition, Tokyo Kasei University, Japan
}

\begin{abstract}
A large number of adult Kazakhs are reported to have hypertension and to exhibit non-dipping blood pressure variations (BPV). The purpose of this study was to determine whether such non-dipping variations also exist in juvenile Kazakhs. This study aimed to clarify the mechanisms responsible for these non-dipping type BPV by examining the autonomic function and physical activity of juvenile Kazakhs. Briefly, we selected clinically healthy Kazakhs who were aged between 9 and 10 years old from the Xinjiang Autonomic Region of the People's Republic of China. All subjects were active and underwent routine physical, blood, and urine examinations. We also performed ambulatory blood pressure monitoring. We assessed the subjects' autonomic function by performing a frequency domain analysis of their heart rate variability (HRV) and monitored the subjects' physical activity with accelerometery. We analysed systolic blood pressure (SBP) variations with the maximum entropy method (MEM). MEM analysis revealed that the SBP variations of the non-dipping juvenile Kazakhs exhibited a 24-hr periodicity (0.04 1 /hr) with a very weak power spectral density. The non-dippers had higher low-frequency/high-frequency (LF/HF) ratios and lower HF/(LF+HF) ratios than the dippers. Neither the 24-hr physical activity scores nor the night-time physical activity scores of the non-dippers and dippers differed significantly. However, the daytime physical activity scores of the non-dippers were lower than those of the dippers. These differences in autonomic function and physical activity might contribute to the generation of weak circadian variations in SBP in juvenile Kazakhs. Thus, these alterations might ultimately lead to the non-dipping SBP variations observed in non-dipping juvenile Kazakhs.
\end{abstract}

KEYWORDS: Blood pressure variation; Non-dipper; Sympathetic; Parasympathetic; Physical activity; Ethnicity; Kazakh; Juvenile

ABBREVIATIONS: ABPM: Ambulatory Blood Pressure Monitoring; BPV: Blood Pressure Variations; BL: Body Length; DBP: Diastolic Blood Pressure; ECG: Electrocardiography; FFT: Fast Fourier Transform; HRV: Heart Rate Variability; HF: High-Frequency; LF: Low-Frequency; MEM: Maximum Entropy Method; PSD: Power Spectral Density; PR: Pulse Rate; PRC: Peoples' Republic of China; SD: Standard Deviation; SBP: Systolic Blood Pressure

Quick Response Code:

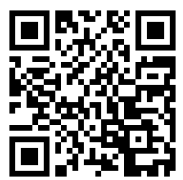

Address for correspondence: Hiroshi Kawamura, Blood Pressure Center, Myojyougakuen (MJG) Cardiovascular Institute, Japan

Received: August 21, $2020 \quad$ Published: October 08, 2020

How to cite this article: Hiroshi K, Yukio O, Yoichi I, Yuji K, Tomohiro N, Hiromi M, Yuhei I. Non-Dipping Blood Pressure Variations in Juvenile Kazakhs are Derived from Increased Sympathetic Activity and Decreased Parasympathetic Activity Particularly Decreased Daytime Physical Activity. 2020 - 2(5) OAJBS.ID.000224. DOI: 10.38125/OAJBS.000224 


\section{INTRODUCTION}

The prevalence of hypertension is high in the northern territory of the Peoples' Republic of China (PRC), where a large number of Kazakhs have been found to display higher blood pressure levels than other minorities [1-4]. We reported that elderly and middleaged Kazakhs in the Balikun area exhibited an increased prevalence of hypertension $[2,3]$. We also found that many of them displayed non-dipping systolic blood pressure (SBP) variations [5,6]. Kazakhs have been reported to exhibit reduced cardiac autonomic functionrelated parasympathetic activity [5,6]. Growing evidence suggests that individuals that exhibit non-dipping blood pressure variations (BPV) are more likely to be older; non-Caucasian; smokers; diabetic; and have target organ damage, such as left ventricular hypertrophy, than those with dipping BPV [7-9]. A few reports have suggested that physical activity plays a role in the mechanisms responsible for non-dipping BPV $[10,11]$. It has been reported that non-dippers exhibit less physical activity in the daytime and greater physical activity in the night-time [12]. The present study focused on whether non-dipping BPV are also seen in juvenile Kazakhs. We hypothesize that non-dipping BPV in juvenile Kazakhs are caused by the same mechanisms as the non-dipping BPV seen in elderly and middle-aged Kazakhs and that the causative mechanism involves autonomic nervous activity and physical activity $[5,6]$. However, no information about the prevalence of non-dipping BPV in juvenile Kazakhs or the mechanism responsible for such variations is available at present. Therefore, we decided to perform ambulatory blood pressure monitoring (ABPM) and a frequency domain analysis of heart rate variability (HRV) and to monitor the physical activity of juvenile Kazakhs. In this report, we present BPV, HRV, and physical activity data collected from two groups of juvenile Kazakhs, those that exhibited dipping-type BPV and those that displayed non-dipping-type BPV.

\section{METHODS AND ETHICS}

This study complied with the ethical principles of the Helsinki Declaration and the guidelines for good clinical practice. Each participating institution received approval for the study from their institutional review board. Written informed consent was obtained from all enrolled subjects.

\section{STUDY POPULATION}

We performed cross-sectional surveillance using a previously described procedure $[3,5,6]$. Briefly, we selected clinically healthy Kazakhs that were aged between 9 and 10 years old from the Balikun region of the Xinjiang Autonomic Region of the People's Republic of China. A total of 40 clinically healthy and active juvenile Kazakhs (males: 21, females: 19) were recruited. All subjects underwent routine physical, blood, and urine examinations as well as chest X-rays. The body mass index was calculated as weight $(\mathrm{kg})$ divided by height. The subjects provided informed consent and were chosen at random based on residence cards from the local government office. All subjects were required to perform their daily physical activities and rest at set times.

\section{4-hr BP Monitoring}

We defined non-dippers as subjects who exhibited a nighttime SBP reduction of less than 10\% compared with their daytime SBP. Based on this definition, the juvenile Kazakh subjects were classified into two groups: 1. juvenile Kazakhs that exhibited dipping BPV (9-10 years old, $n=29$ ) and 2. juvenile Kazakhs that displayed non-dipping BPV (9-10 years old, $n=11)$. We used 14 automatic sociometric devices to record the subjects' SBP, diastolic blood pressure (DBP), and pulse rate (PR), as described previously (3). Meals were taken between the hours of 06:30 and 07:30, 11:00 and 12:00, and 17:30 and 18:30. Each subject was given a diary to record their daily physical activity, including the times at which they ate meals, slept, and micturated. All of the 24-hr measurements, as well as the subjects' daytime and night-time BP levels, are shown as mean values. The 24-hr measurements were averaged at 30min intervals in order to obtain mean and standard deviation (SD) values. Abnormal SBP values were excluded based on previously reported criteria [3].

\section{4-hr Holter ECG Recording}

The subjects were monitored for $24 \mathrm{hr}$ with a Holter electrocardiography (ECG) recording system, with the data collection starting $2 \mathrm{hr}$ after the initiation of the ABPM. The ECG data were recorded at a sampling frequency of $256 \mathrm{~Hz}$ and stored on a tape recorder, as described previously $[5,6]$. We performed fast Fourier transform (FFT) analysis every $60 \mathrm{~s}$. We selected ECG recordings with consistent sinus rhythms for analysis, as described previously $[5,6]$.

\section{Frequency Domain Analysis}

We monitored the subjects' autonomic nervous system activity by performing a frequency domain analysis of their HRV, which was recorded using ECG, over $24 \mathrm{hr}$. The subjects' HRV was calculated using the FFT for each segment, and the following frequency domain variables were calculated: low-frequency (LF) (0.04-0.15 $\mathrm{Hz}$ ) power $\left(\mathrm{ms}^{2} / \mathrm{Hz}\right)$, high-frequency $(\mathrm{HF})(0.15-0.40 \mathrm{~Hz})$ power $\left(\mathrm{ms}^{2} / \mathrm{Hz}\right)$, the $\mathrm{LF} / \mathrm{HF}$ ratio, and the $\mathrm{HF} /(\mathrm{HF}+\mathrm{LF})$ ratio. We analysed the data every minute.

\section{Spectral Analysis of SBP Variation}

After averaging the observed data (every $30 \mathrm{~min}$ ), the variation in the subjects' SBP was analysed using the maximum entropy method (MEM), as described previously [6]. The main analysis was based on the MEM and the non-linear least squares method.

\section{Physical Activity}

We monitored the subjects' physical activity (physical activity scores) with accelerometery (Life-Corder, Suzuken Co., Tokyo, Japan); i.e., the intensity of the subjects' physical activity was graded on a 10-point scale every $5 \mathrm{~min}$.

\section{STATISTICAL ANALYSIS}

Data obtained using parametric analyses are expressed as mean \pm SD values, and those obtained using non-parametric analyses are shown as median (Q1, Q3) values. All conventional statistical calculations were performed using the Statistical Analysis System (SAS Institute Inc., SAS Campus Drive, Cary, NC). Inter-group comparisons were carried using two-way analysis of variance (ANOVA) or non-parametric analyses, such as the Mann-Whitney $\mathrm{U}$-test or F-test. $P$-values of $<0.05$ were statistically significant.

\section{RESULTS}

\section{Physiological Characteristics of Juvenile Kazakhs}

As shown in Table 1, the dippers and non-dippers accounted for $72 \%(n=29)$ and $28 \%(n=11)$ of the subjects, respectively. The physiological characteristics and mean 24-hr SBP, DBP, and PR values of the dippers and non-dippers did not differ significantly (n.s., Table 1). However, the SBP of the non-dippers was lower 
during the daytime and higher during the night-time than those of the dippers (Table 1). The DBP of the non-dippers was lower during the daytime and higher during the night-time than those of the dippers. The PR of the dippers and non-dippers did not differ significantly at any timepoint.

Table 1: Physiological characteristics: H: Height; SBP: Systolic Blood Pressure; DBP: Diastolic Blood Pressure; PR: Pulse Rate; Mean (SD); n.a: Not Applicable; P-values were calculated using the Kruskal-Wallis test.

\begin{tabular}{|c|c|c|c|}
\hline Number & Dippers & Non-dippers & p-value \\
\hline$\%$ & 29 & 11 & n.a. \\
\hline Sex ratio & 72 & 28 & 0.87961 \\
\hline Age (Y/O) & $1.5 \pm 0.5$ & $1.5 \pm 0.5$ & 0.53325 \\
\hline BL (cm) & $9.7 \pm 0.5$ & $9.8 \pm 0.4$ & 0.11624 \\
\hline $24 \mathrm{hr}$ SBP (mmHg) & $139 \pm 7$ & $140 \pm 7$ & 0.545567 \\
\hline $24 \mathrm{hr}$ DBP (mmHg) & $116 \pm 8$ & $115 \pm 5$ & 0.60923 \\
\hline $24 \mathrm{hr}$ PR (beats/min) & $69 \pm 5$ & $69 \pm 3$ & 0.86882 \\
\hline Daytime SBP (mmHg) & $79 \pm 6$ & $79 \pm 9$ & 0.02172 \\
\hline Daytime DBP (mmHg) & $123 \pm 10$ & $117 \pm 5$ & 0.02663 \\
\hline Daytime PR (beats/min) & $75 \pm 7$ & $72 \pm 3$ & 0.76261 \\
\hline Night-time SBP (mmHg) & $81 \pm 7$ & $79 \pm 9$ & 0.00041 \\
\hline Night-time DBP (mmHg) & $98 \pm 7$ & $63 \pm 7$ & 0.00599 \\
\hline Night-time PR (beats/min) & $56 \pm 6$ & & \\
\hline
\end{tabular}

SBP Variations of the Dipping and Non-Dipping Juvenile Kazakhs

The SBP variations of the dippers exhibited clear circadian variations, with their SBP rising during the daytime and falling at night-time (left and right panels, Figure 1). As expected, the nondippers demonstrated less marked circadian variations in their SBP than the dippers (left and right panels, Figure 1). The SBP levels of the dippers and non-dippers differed significantly at many timepoints (dippers vs. non-dippers: $96 \pm 11$ vs. $116 \pm 23$ at 0:00; $97 \pm 8$ vs. $117 \pm 28$ at $00: 30 ; 93 \pm 10 v s .102 \pm 9$ at $01: 30 ; 93 \pm 10 v s$. $114 \pm 23$ at $02: 00 ; 92 \pm 9$ vs. $103 \pm 10$ at 03:30; $93 \pm 7 v s .106 \pm 19$ at 4:00; $94 \pm 11$ vs. $105 \pm 8$ at 05:00; $96 \pm 9$ vs. $102 \pm 8$ at 06:30; $141 \pm$ 33 vs. $125 \pm 13$ at $12: 00 ; 132 \pm 28$ vs. $115 \pm 15$ at $13: 30 ; 127 \pm 23$ vs. $103 \pm 17$ at $18: 00 ; 137 \pm 31$ vs. $117 \pm 25$ at 18:30; $124 \pm 24$ vs. 106 \pm 17 at $22: 30$; all $\mathrm{P}<0.05$ ).

\section{Circadian Variations of SAP in juvenile Kazakhs}

Dippers

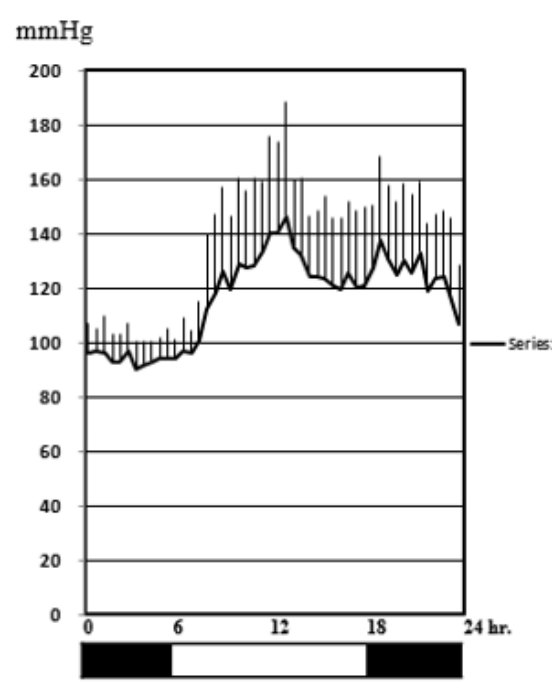

Non-dippers

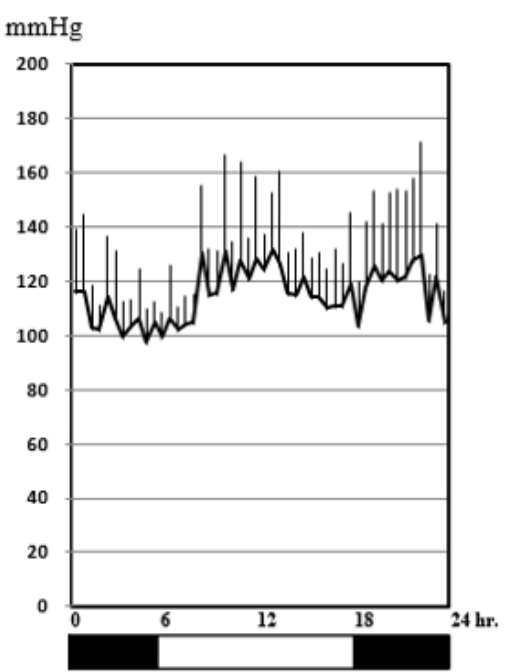

Figure 1: Juvenile Kazaks exhibited clear circadian variations in their SBP, with their SBP rising during the daytime and falling at night-time (left and right panels). However, these circadian variations were less clear in the nondipping juvenile Kazakhs, as shown in the right panel. 
Spectral Analysis of SBP Variations in Juvenile Kazakhs

We analysed the variations in the mean SBP values of the juvenile Kazakhs using MEM, which revealed that the SBP variations of the dippers and non-dippers displayed a 24-hr periodicity (approximately $0.041 / \mathrm{hr}$ ) and ultradian variations. The 24-hr periodicity of the dippers' SBP values demonstrated a greater power spectral density (PSD) than that of the non-dippers (upper panel, Figure 2). In fact, the SBP variations of the non-dippers exhibited a 24-hr periodicity with a very weak PSD (lower panel, Figure 2).

\section{Spectral density of SAP in juvenile Kazakhs}
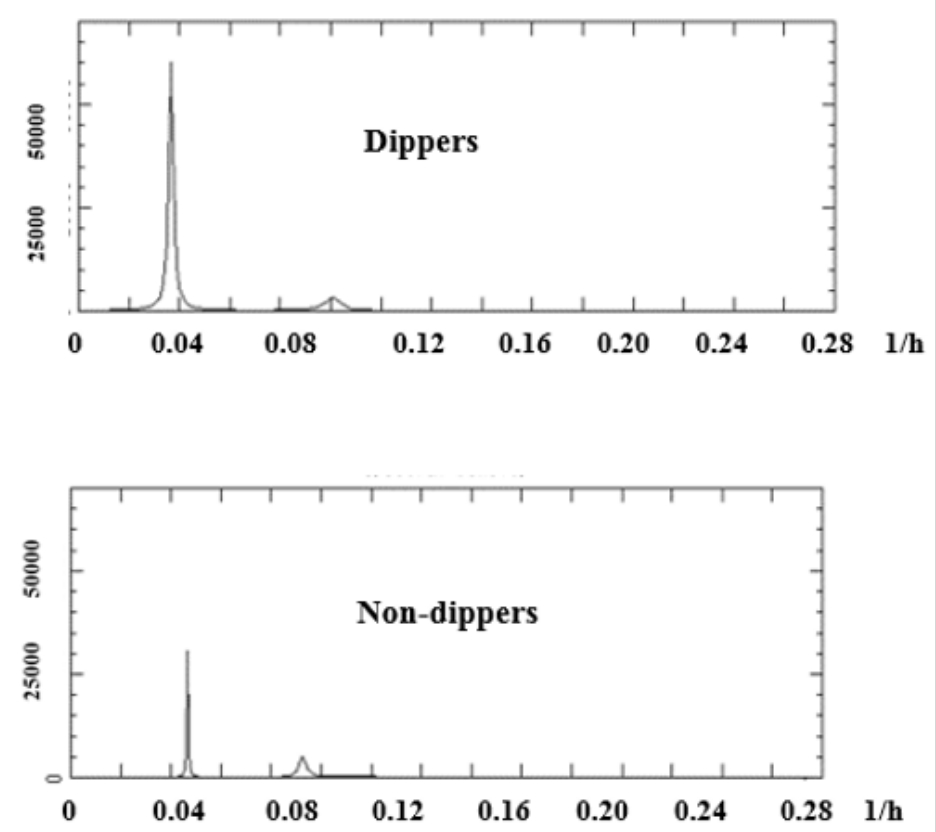

Figure 2: The SBP variations of the dipping juvenile Kazakhs displayed a 24-hr periodicity (close to $0.041 / \mathrm{h}$, and ultradian variations, upper panel) and a greater PSD than that of non-dippers (lower panel). In contrast to those of the dippers, the SBP variations of the non-dippers exhibited a 24-hr periodicity with a very weak PSD and ultradian variations (lower panel).

\section{Circadian Variations in the LF/HF Ratios of Juvenile Kazakhs}

We detected circadian variations in the LF/HF ratios of the juvenile Kazakhs (Figure 3). The LF/HF ratio rose during the daytime (white bar, Figure 3) and fell during the night-time (black bar, Figure 3) in both the dippers and non-dippers (left and right panels, Figure 3). The non-dipping juvenile Kazakhs exhibited higher LF/HF ratios than the dipping juvenile Kazakhs at 36 timepoints $(1: 05,1: 10,1: 45,2: 30,3: 35,3: 45,4: 15,4: 20,6: 10$, $6: 45,6: 55,7: 05,7: 55,8: 20,8: 30,8: 35,8: 55,9: 00,9: 15,9: 30,10: 10$, $10: 20,13: 10,13: 25,13: 30,14: 15,14: 50,15: 25,15: 45,16: 10,16: 30$, 19:25, 19:45, 21:45, 22:00, 22:40; all $P<0.0497$ ).

\section{Circadian Variations in the $\mathrm{HF} /(\mathrm{LF}+\mathrm{HF})$ Ratios of} Juvenile Kazakhs

We also detected circadian variations in the $\mathrm{HF} /(\mathrm{LF}+\mathrm{HF})$ ratios of the juvenile Kazakhs (Figure 4). In contrast to the LF/HF ratio, the $\mathrm{HF} /(\mathrm{LF}+\mathrm{HF})$ ratio fell during the daytime (white bar, Figure 4) and rose during the night-time (black bar, Figure 4) in both the dippers and non-dippers. The HF/(LF+HF) ratios of the nondipping juvenile Kazakhs were lower than those of the dipping juvenile Kazakhs at 35 timepoints $(1: 10,1: 45,2: 30$, 3:35, 3:45, 4:15, $4: 20,6: 10,6: 45,6: 55,7: 05,7: 55,8: 20,8: 30,8: 35,8: 55,9: 00,9: 15$, $9: 30,10: 10,10: 20,13: 10,13: 25,13: 30,14: 15,14: 50,15: 25,15: 45$,
16:10, 16:30, 19:25, 19:45, 21:45, 22:00, 22:40; all $P<0.0497)$.

\section{Circadian Variations in the Physical Activity Scores of Juvenile Kazakhs}

We found that the physical activity scores of the juvenile Kazakhs, which reflected the intensity of their daily physical activity, displayed circadian variations (Figure 5). The physical activity scores of both the dippers and non-dippers rose during the daytime (white bar, Figure 5) and fell during the night-time (black bar, Figure 5). The physical activity scores of the dippers and nondippers differed significantly at many timepoints (dippers vs. nondippers: $1.5 \pm 0.0 .8$ vs. $1.0 \pm 0.5$ at 9:00; $1.7 \pm 1.3$ vs. $1.1 \pm 0.6$ at $10: 00 ; 1.9 \pm 0.9$ vs. $0.9 \pm 0.4$ at $13: 10 ; 1.7 \pm 0.9$ vs. $0.9 \pm 0.5$ at $13: 50$; $1.9 \pm 1.1$ vs. $1.0 \pm 0.5$ at $14: 10 ; 1.5 \pm 1.2$ vs. $0.7 \pm 0.4$ at $15: 00 ; 1.2 \pm$ 1.0 vs. $0.5 \pm 0.4$ at $15: 10 ; 1.9 \pm 1.1$ vs. $0.8 \pm 0.5$ at $17: 50 ; 1.7 \pm 0.8$ vs. $1.2 \pm 0.7$ at $18: 50 ; 1.0 \pm 0.7$ vs. $0.7 \pm 0.4$ at $21: 40$, all $P<0.05$ ).

\section{Median LF/HF Ratios, HF/(LF+HF) Ratios and Physical Activity Scores}

As shown in Figure 6, the non-dippers' LF/HF ratios (24-hr, daytime, and night-time) were higher than those of the dippers (dippers vs. non-dippers: 24-hr: median: 0.43, Q1: 0.37, Q3: 0.57 vs. median: 0.51, Q1: 0.44, Q3: 0.61, Mann-Whitney U-test: $P=0.0001$, $F$-test: $P=0.00860$ [left panel, Figure 6]; daytime: median: 0.52, Q1: 0.39, Q3: 0.59 vs. median: 0.56, Q1: 0.48, Q3: 0.66, Mann-Whitney 
U-test: $P=0.0001$, F-test: $P=0.0001$ [middle panel, Figure 6]; nighttime: median: $0.37, \mathrm{Q} 1: 0.34, \mathrm{Q} 3: 0.39$ vs. median: $0.42, \mathrm{Q} 1: 0.38$,

Q3: $0.46, F$-test: $P=0.0001$, Mann-Whitney U-test: $P=0.0001$ [right panel, Figure 6]).

\section{Circadian Variations of LF/HF in juvenile Kazakhs}
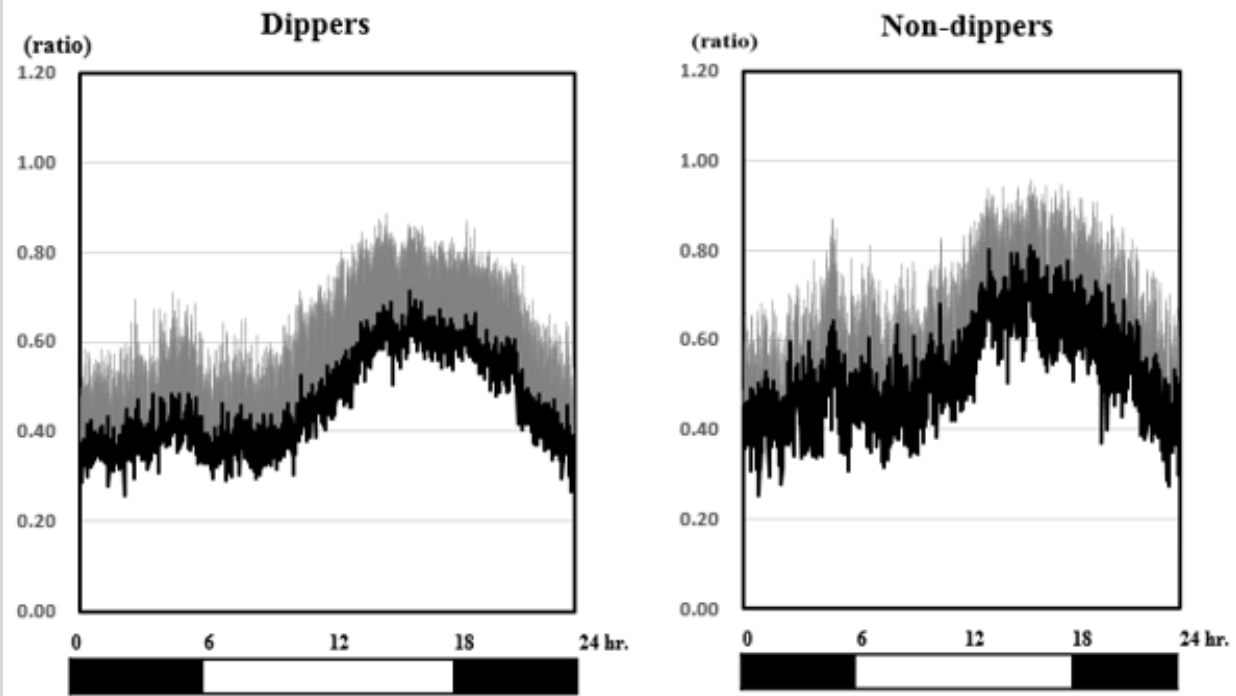

Figure 3: The LF/HF ratio rose during the daytime (light bar) and fell during the night-time (dark bar) in both the dipping and non-dipping juvenile Kazakhs.

\section{Circadian Variations of $\mathrm{HF} /(\mathrm{LF}+\mathrm{HF})$ in juvenile Kazakhs}
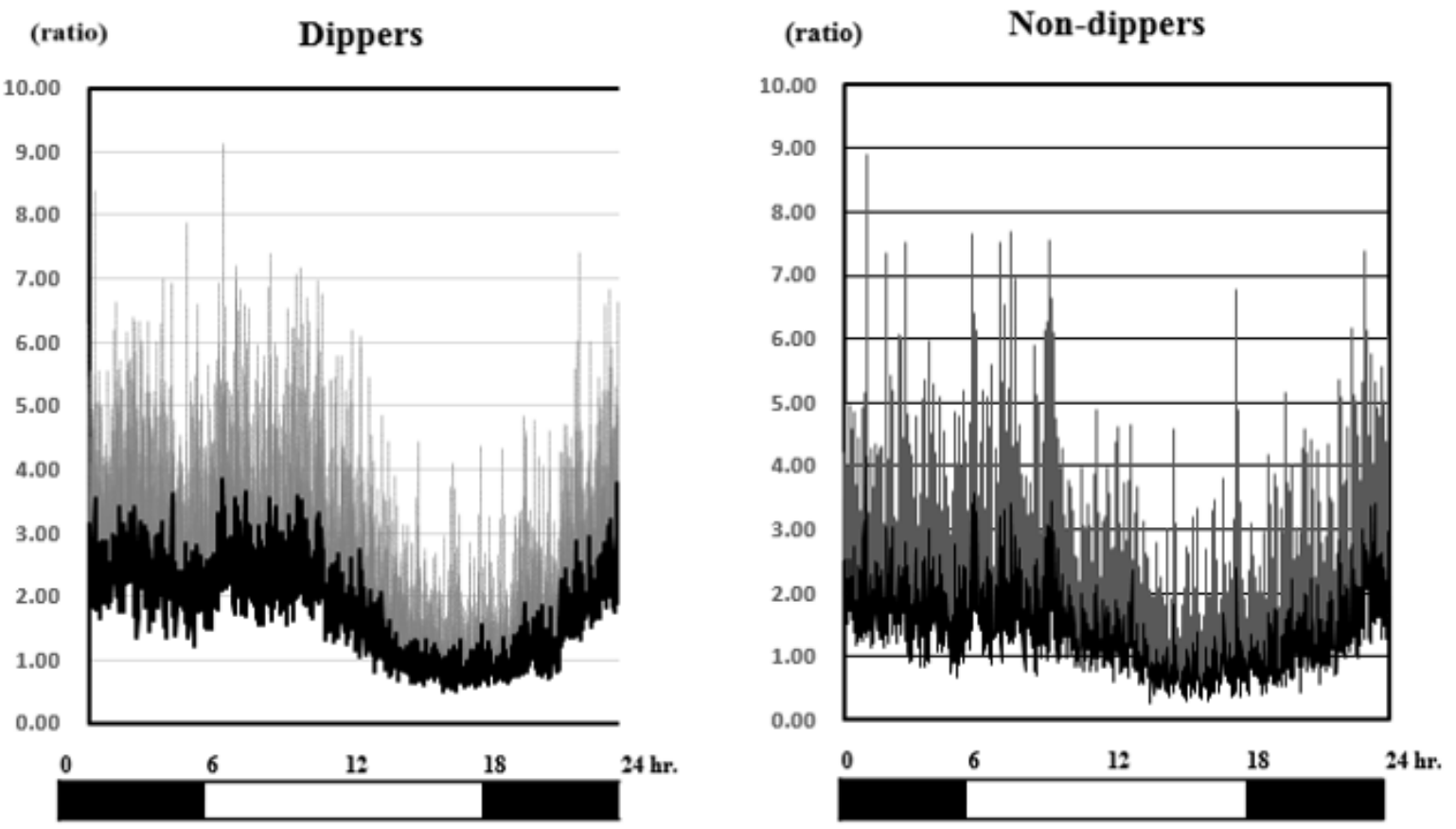

Figure 4: TThe HF/(LF+HF) ratios fell during the daytime (light bar) and rose during the night-time (dark bar) in both the dippers and non-dippers. 


\section{Circadian Variations of Physical Activity in juvenile Kazakhs}
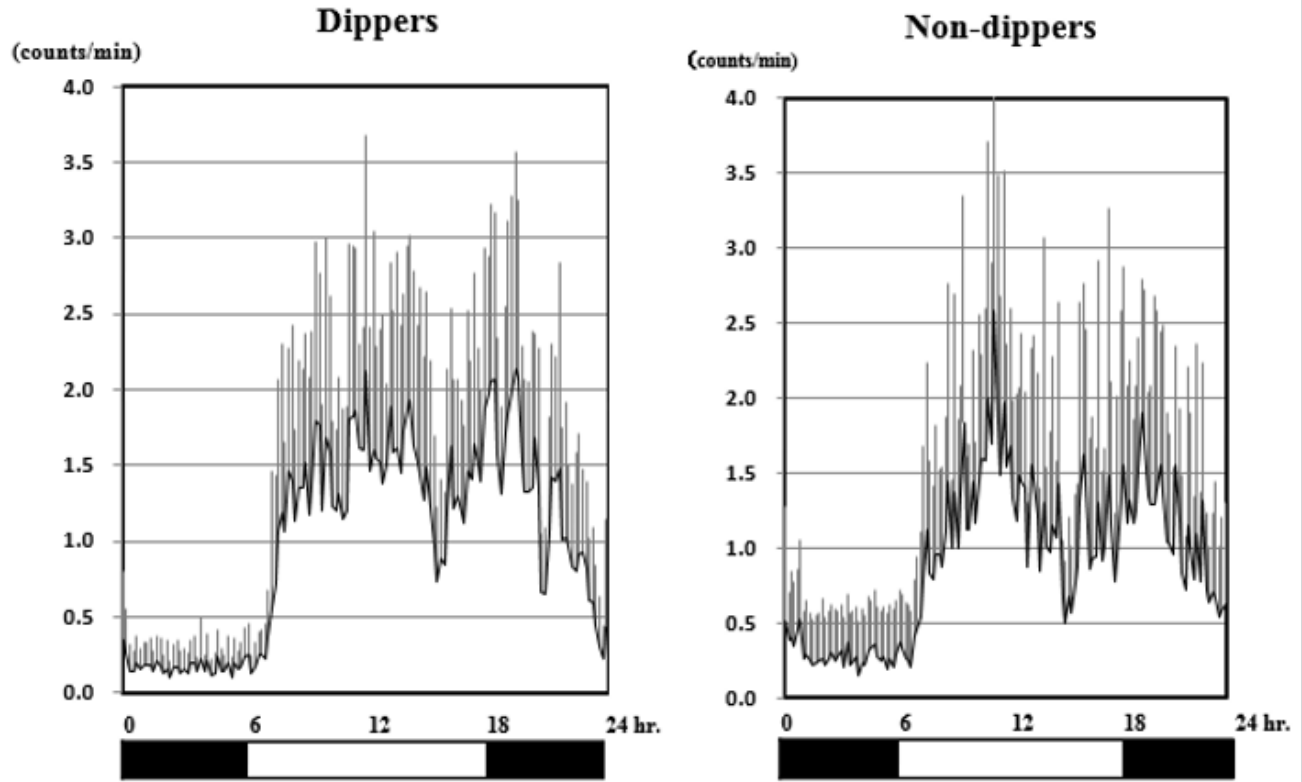

Figure 5: The physical activity scores of both the dippers and non-dippers rose during the daytime (light bar) and fell during the night-time (dark bar).

\section{Median (Q1,Q3) of LF/HF in juvenile Kazakhs}

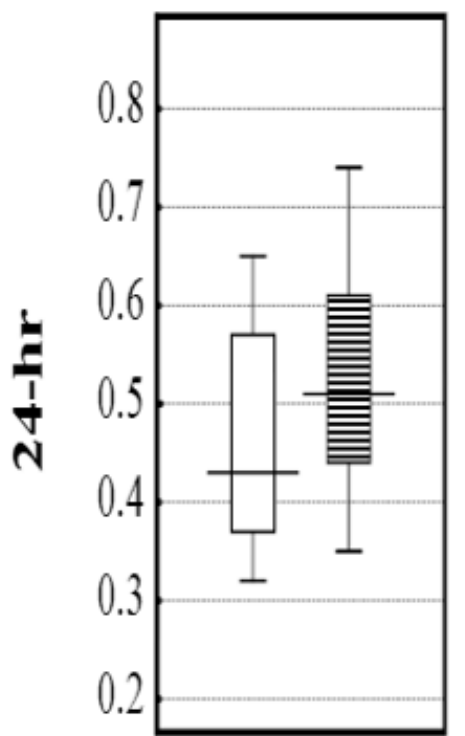

Dippers Non-dippers

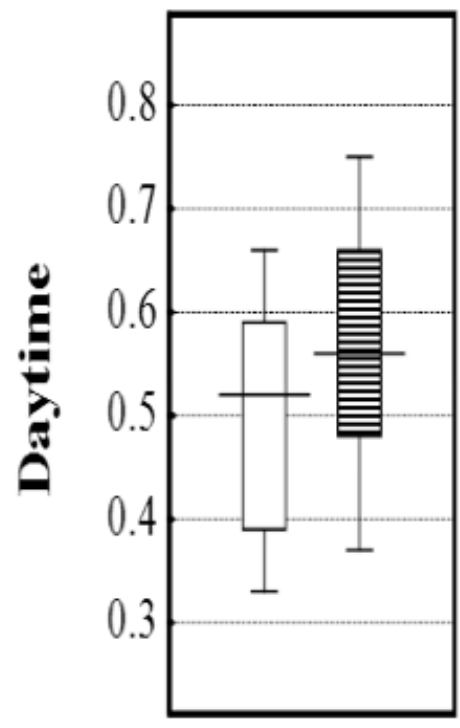

Dippers Non-dippers

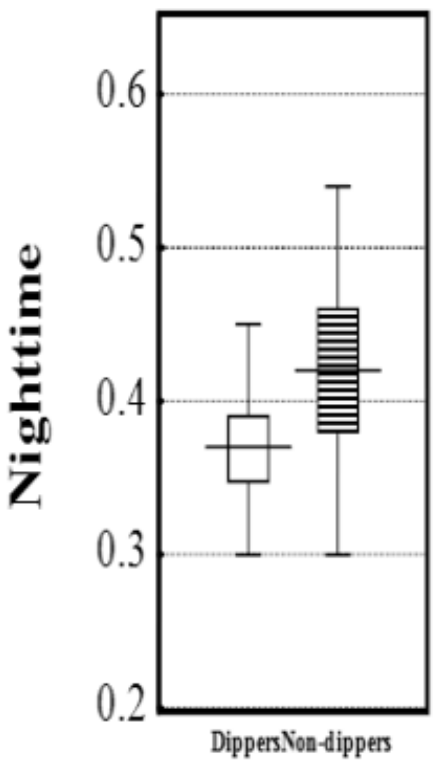

Figure 6: Significant differences between the LF/HF ratios of the dippers and non-dippers were observed over $24 \mathrm{hr}$ and during the daytime and night-time (dippers vs. non-dippers; $24 \mathrm{hr}$, daytime, and night-time, all F-test, $P<0.00001)$.

As shown in Figure 7, the non-dippers' HF/(LF+HF) ratios were lower than those of the dippers (dippers vs. non-dippers: 24-hr: median: 1.87, Q1: 1.11, Q3: 2.34 vs. median: 1.30, Q1: 0.92, Q3: 1.75, F-test: $P=0.0001$, Mann-Whitney U-test: $P=0.0001$ [left panel, Figure 7]; daytime: median: 1.47, Q1: 0.93, Q3: 2.18 vs. median:
1.10, Q1: 0.78, Q3: 1.53, F-test: $P=0.0001$, Mann-Whitney U-test: $P=0.0001$ [middle panel, Figure 7]; night-time: median: 2.37, Q1: 2.12, Q3: 2.64 vs. median: 1.83, Q1: 1.51, Q3: 2.18, F-test: $P=0.0009$, Mann-Whitney U-test: $P=0.0001$ [right panel, Figure 7]). 


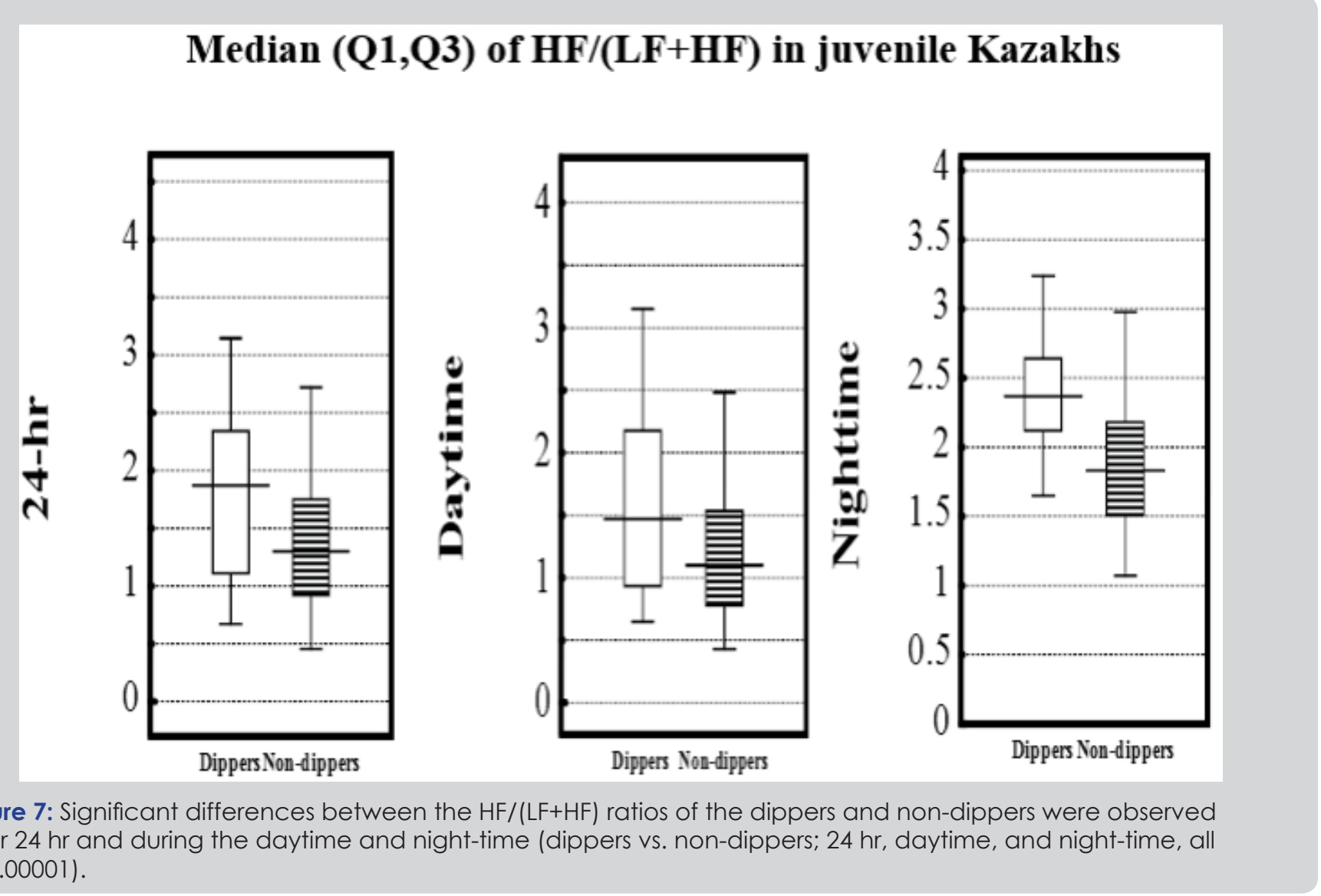

As shown in Figure 8, the 24-hr and night-time physical activity scores of the dippers and non-dippers did not differ significantly (dippers vs. non-dippers: 24-hr: median: 1.20, Q1: 0.20, Q3: 1.50 vs. median: 0.90, Q1: 0.10, Q3: $1.30, F$-test: $P=0.0050$, Mann-Whitney U-test $P=0.4368$ [left panel, Figure 8]; night-time: median: 0.42, Q1: $0.21, \mathrm{Q} 3: 0.75$ vs. median: $0.40, \mathrm{Q} 1: 0.20, \mathrm{Q}: 0.78, F$-test: $P=0.4850$,
Mann-Whitney U-test: $P=0.8270$ [right panel, Figure 8]). However, the daytime physical activity scores of the non-dippers were lower than those of the dippers (dippers vs. non-dippers: median: 1.27, Q1: 1.06, Q3: 1.38 vs. median: 1.00, Q1: 0.80, Q3: 1.20, F-test: $P=0.0733$, Mann-Whitney U-test: $P=0.0001$ [middle panel Figure 8]).

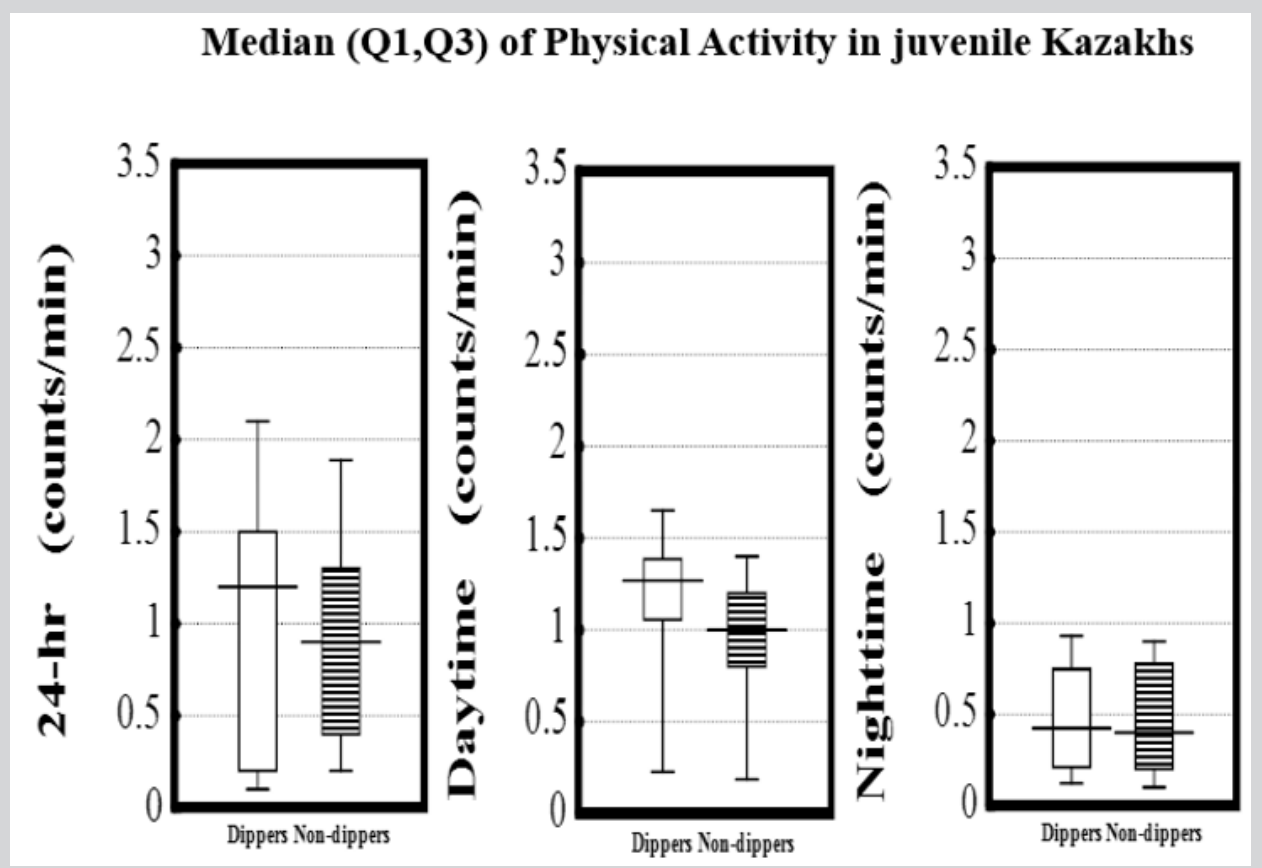

Figure 8: The physical activity scores of the dipping and non-dipping juvenile Kazakhs only differed during the daytime (dippers vs. non-dippers; daytime, $P<0.0001$ ). 


\section{DISCUSSION}

Previously, we reported that many middle-aged and elderly Kazaks were hypertensive and non-dippers $[2,3,5,6]$. As shown in Table 1 , in the present study dippers and non-dippers accounted for $72 \%$ and $28 \%$ of the subjects, respectively. A previous study reported that SBP was related to height in both males and females [13]. However, our study did not detect any difference in height between the dippers and non-dippers, which also agrees with the findings of another study [14]. Therefore, the differences between the BP patterns of the dippers and non-dippers might not have resulted from differences in physiological characteristics.

\section{Circadian Variations in SBP in Juvenile Kazakhs}

Our previous results suggested that $52 \%$ of middle-aged Kazakhs are non-dippers, but also that such individuals exhibit similar physiological characteristics to dippers. In the current study, the SBP of both the dippers and non-dippers exhibited daytime peaks and midnight troughs (left panel, Figure 1), and their SBP variations displayed circadian rhythms. However, the SBP variations of the non-dippers exhibited weaker circadian rhythms (right panel, Figure 2). It was previously demonstrated that the likelihood of becoming a non-dipper increases with age $[15,16]$. However, in the present study, the living conditions of the dippers and non-dippers were similar, and the timing of physical activities, such as eating, rising, and sleeping, was also standardized. To confirm when physical activities occurred, we examined the diaries of all the subjects.

\section{Circadian Variations in the LF/HF ratios, HF/(HF+LF) Ratios and Physical Activity Scores of Juvenile Kazakhs}

We consider that frequency domain analysis of HRV is the most useful technique for comparing dippers and non-dippers. In the present study, the subjects' LF/HF ratios displayed daytime peaks and midnight troughs (Figure 3), while their $\mathrm{HF} /(\mathrm{HF}+\mathrm{LF})$ ratios exhibited a midnight peak and a daytime trough (Figure 4). Thus, the subjects' $\mathrm{LF} / \mathrm{HF}$ and $\mathrm{HF} /(\mathrm{HF}+\mathrm{LF})$ ratios both displayed circadian variations. The SBP variations of the non-dippers exhibited a circadian rhythm with a weak PSD (Figure 2); thus, the cardiac autonomic nervous system might be at least partially responsible for generating $\mathrm{LF} / \mathrm{HF}$ and $\mathrm{HF} /(\mathrm{LF}+\mathrm{HF})$ ratio variations. It has been reported that non-dippers demonstrate relatively high sympathetic nervous system activity during the night-time [18]. Our findings of high sympathetic activity and low parasympathetic and physical activity in the non-dippers agree with these reports. They could also explain the high night-time SBP of the non-dippers. In contrast, another study detected low LF values in non-dippers [19]. However, the latter study examined 24-hr LF/HF ratios instead of separately analysing daytime and night-time LF/HF ratios. If they had measured LF/HF separately during the daytime and night-time, their results might have been different due to the effects of differences in physical activity. Therefore, we consider that this might explain the discrepancy between their results and ours. Furthermore, we postulate that the non-dipping pattern results from the generation of weak circadian variations caused by disturbances of cardiac autonomic function.

Similar to the subjects' LF/HF ratios, their physical activity scores displayed daytime peaks and midnight troughs (Figure 5). No marked differences in circadian rhythms were detected between the dippers and non-dippers. However, the non-dippers exhibited lower daytime physical activity scores than the dippers (middle panel, Figure 8). On the other hand, no differences were noted between the 24-hr or night-time physical activity scores of the dippers and non-dippers (left and right panels, Figure 8). This indicates that the non-dippers performed less physical activity during the daytime than the dippers. Non-dippers were reported to exhibit smaller increases in their plasma norepinephrine levels than dippers during exercise [10]. This finding would also explain the lower SBP values seen in the non-dippers during the daytime (Figure 1). Nocturnal behavioural activity, such as moving, walking, micturition, wakefulness, and so forth, might lead to the development of a non-dipping status. Based on the entries in the subjects' diaries, no differences were noted between the nocturnal behaviour or quality of sleep of the dippers and non-dippers. Therefore, neither nocturnal urination nor sleep quality appear to be involved in the appearance of non-dipping BPV in this group of juvenile Kazakhs. In field surveillance studies, similar to the current study, it is difficult to assess sleep quality using polysomnography alone. However, our findings suggest that the development of a non-dipping BPV pattern could be caused, at least in part, by the generation of weak circadian rhythms due to insufficient cardiac autonomic function and decreased physical activity during the daytime. Among juvenile Kazakhs, the number of non-dippers was smaller than the number of dippers. This difference might have been age-related, as we reported in our previous studies $[2,3,5,6]$. Based on the findings of the latter studies and the present study, the number of non-dippers seems to increase with age.

In summary, among juvenile Kazakhs the number of non-dippers was smaller than the number of dippers. The non-dipping juvenile Kazakhs exhibited higher $\mathrm{LF} / \mathrm{HF}$ and lower $\mathrm{HF} /(\mathrm{LF}+\mathrm{HF})$ ratios than the dippers. Furthermore, the non-dipping juvenile Kazakhs also performed less physical activity than the dippers during the daytime. These differences in cardiac autonomic functions and physical activity might contribute to the generation of weak circadian rhythms in SBP, and thus, lead to the non-dipping SBP variations observed in non-dipping juvenile Kazakhs.

\section{STUDY LIMITATIONS}

Kazakhs are nomadic. For this reason, it was not possible to obtain a sample size that was large enough to enable separate statistical analyses of normotensives and hypertensives, particularly as most of the juvenile Kazakhs were normotensive. Therefore, we did not divide the juvenile Kazakhs into hypertensives and normotensives. Another limitation of our study was that the numbers of dippers $(n=29)$ and non-dippers $(n=11)$ were not identical because most of the juveniles were dippers. While this might have reduced the statistical power of the study, small inter-group variations were seen in the mean and median values of the majority of the examined parameters. Therefore, we consider that the study had sufficient statistical power to detect significant differences in most of the variables. Furthermore, as the study population was notlarge enough to allow it to be divided into normotensives and hypertensives, we could not examine the mechanisms responsible for non-dipping BPV separately in these groups. We speculate that the mechanism responsible for non-dipping is the same in normotensives and hypertensives since blood pressure gradually increases with age. Another limitation of this particular type of study is the necessity to assign subjects to a group based on their physical activity (dipper pattern) rather than at random. While the self-selecting nature of this cross-sectional study was unavoidable, it proved to be the study's main limitation, as unknown and unstudied variables could have been responsible for differences in the subjects' BPV patterns instead of the mechanisms we specifically explored and discussed. Therefore, future prospective studies with predefined endpoints 
will need to be undertaken. The final limitation of the current study is that the period of BP monitoring was only $24 \mathrm{hr}$. This might have been too brief to allow a thorough and reliable evaluation of BPV or the elimination of extraneous influences, such as the novelty of the study setting. However, conducting a large-scale ABPM field study over a period longer than $24 \mathrm{hr}$ in such a remote location is not practical. In any case, we consider that none of the abovementioned limitations significantly influenced any of the conclusions of the present study.

\section{PERSPECTIVE}

In future, we hope to conduct prospective studies that will enable us to compare the survival rates of juvenile dippers and non-dippers after they reach adulthood. We hypothesize that in the Kazakh population, dippers will have longer lifespans than non-dippers. In addition, further chronobiological studies of this minority population in the PRC might be a valuable way of identifying physical activity lifestyle patterns that are associated with higher overall survival rates.

\section{CONCLUSION}

Non-dipping BPV are seen in juvenile Kazakhs. However, the number of non-dippers was smaller than the number of dippers. Non-dipping juvenile Kazakhs demonstrated SBP variations that exhibited a 24-hr periodicity with a weak PSD. Their cardiac sympathetic nerve activity was increased, and their cardiac parasympathetic nerve activity was decreased. Furthermore, the non-dipping juvenile Kazakhs performed less physical activity than the dipping juvenile Kazakhs during the daytime.

\section{ACKNOWLEDGMENT}

We thank Ms. Yoshiko Aikawa and Noriko Tsujiguchi for their assistance with the preparation of this manuscript.

\section{REFERENCES}

1. Wu X, Duan X, Gu D (1995) Prevalence of hypertension and its trends in Chinese populations. Int J Cardiol 52(1): 39-44.

2. Mu YM, Ozawa Y, Wang SZ (2006) Ultrasonographic study of carotid artery structural changes with natural longevity. Circ J 64(4): 243-248.

3. Kawamura H, Jumabay M, Mitsubayashi H (2000) 24-hrblood Pressure in Uygur, Kazakhs and Han elderly subjects in China. Hypertens Res 23(2): 177-185.

4. Liu L, Liu L, Ding Y (2001) Ethnic and environmental differences in various markers of dietary intake and blood pressure among Chinese Han and three other minority peoples of China: Results from the WHO cardiovascular diseases and alimentary comparison (CARDIAC) study. Hypertens Res 24(3): 315-322.
5. Kawamura H, Mitsubayashi H, Izumi Y (2009) Analysis of heart rate variability amongst non-dipper Kazakh, Xinjiang, China. Jpn J Clin Physiol 39(3): 155-162.

6. Kawamura H, Ozawa H, Izumi Y (2016) Non-dipping blood pressure variations in adult Kazakhs are derived from increased daytime physical activity and increased sympathetic activity. Clin Exp Hypertens 38(2): 194-202.

7. Hermida PC, Ayaia DF, Mojon A, Fernandez JR (2013) Blunted sleeptime relative blood pressure decline increases cardiovascular risk independent of blood pressure level-the "normotensive non-dipper" paradox. Chronobiol Int 30(1-2): 87-98.

8. Verdecchia P, Schillaci G, Guerrieri M (1990) Circadian blood pressure change sand left ventricular hypertrophy in essential hypertension. Circulation 81(2): 528-536.

9. Brotman DJ, Davidson MB, Boumitri M, Vidt DG (2008) Impaired diurnal blood pressure variation and all-cause mortality. Am J Hypertens 21(1): 92-97.

10. Arita M, Minami E, Nakamura C (1996) Role of sympathetic nervous system in the nocturnal fall in blood pressure. Hypertens Res 19(3): 195-200.

11. Kario K, Schwartz J, Pickering TG (1999) Ambulatory physical activity as a determinant of diurnal blood pressure variation. Hypertens 34: 685691.

12. Leary AC, MacDonald TM, Murphy MB (2000) Physical activity level is an independent predictor of the diurnal variation in blood pressure. J Hypertens 18(4): 405-410.

13. O’Sullvan JJ, Derrick G, Griggs P, Foxall R, Wren C (1999) Ambulatory blood pressure in schoolchildren. Arch Dis Child 80(6): 529-532.

14. Krzych LJ, Szydlowski L (2009) Determinants of inappropriate circadian blood pressure variability in children with essential hypertension Can J Cardiol 25(1): e13-e16.

15. Peres-Lloret S, Aguirre AG, Cardinali DP, Toblli JE (2004) Disruption of ultradianan d circadian rhythms of blood pressure in non-dipper hypertensive patients. Hypertension 44(3): 311-315.

16. Staessen JA, Bienaszewski L, O’Brien E (1997) Nocturnal blood pressure fall on ambulatory blood pressure monitoring in a large international data base. Hypertension 29: 30-39.

17. Ebata H, Hojo Y, Ikeda U (1995) Differential effects of an a1-blocker (doxazosin) on dirnal blood pressure variation in dipper and non-dipper type hypertension. Hypertens Res 18(2): 125-130.

18. Nakano Y, Oshima T, Ozomno R (2001) Non-dippper phenomena on in essential hypertension is related to blunted nocturnal rise and fall of sympathetic-vagal nervous physical activity and progress in retinopathy. Autonomic Neuroscience 88(3): 181-186.

19. Ragot S, Harpin D, Siche JP, Ingrand P (1999) Autonomic nervous system physical activity in dipper and non-dipper essential hypertensive patients. What about sex differences? J Hypertens 17(12): 1805-1811. 